\title{
FUGUE AND VARIATIONS ON SOME THEMES IN ART AND SCIENCE
}

\author{
Gordana Novakovic \\ Artist-in-residence, Computer Science Department \\ University College London \\ Malet Place \\ London WC1E 6BT \\ UK \\ Gordana.Novakovic@,cs.ucl.ac.uk \\ http://gordananovakovic.com
}

\begin{abstract}
This paper describes the development over several years of Fugue, an art| science audio-visual piece inspired by the human immune system. It has been presented in a number of different contexts - as an artwork, as an aid to the public understanding of science, and as a potential tool for scientists - and it is still under development. Stimulated by the response of some participants to the interactive and immersive version of Fugue, by recent discoveries in the field of neuroplasticity, and by contemporary analysis and criticism of some adverse effects of the digital revolution, a possible new category of art, neuroplastic art, is identified and briefly discussed.
\end{abstract}

\section{PRELUDE}

I began my professional life as a painter, but when computers became available from 1984 or so, I began to use them in various ways as tools and media. I made my first computer-controlled interactive piece in 1994 (see figure 1), and was immediately struck by the powerful and unexpected responses shown by some participants. This stimulated my interest in perception, and in the psychological aspects of the complex experience of interactivity, but also introduced me to the broader framework of the digital revolution, media criticism, sociology, philosophy, and so on. In 2001, several participants in my large-scale interactive installation Infonoise [13] (figure 2) showed puzzling signs of distorted consciousness, and so I began to explore the transdisciplinary field of consciousness studies [10]. It was against this background that I began work on another large-scale interactive piece, Fugue, and once again found myself asking questions about the strange effects of digital interactive technology.

\section{FUGUE}

An Arts Council England Individual Grant in 2004 brought modest funding for Algorithmica, originally titled City Portrait. Firmly grounded in research, it aimed to critically address the form of the mass-media industry. A spontaneous, non-tactile interaction was to be based on the biological principles of interaction among cells, and a game-based software architecture would operate within the set of a 3D London Tube map. Dr Peter Bentley, a UCL-based expert on computational models of the human immune system, joined the project, along with Anthony Ruto, an expert on 3D modelling also from UCL, and my long-term collaborator Rainer Linz, the Australian 


\section{EVA 2009 London Conference 6-8 July \\ Gordana Novakovic}

new music composer. We agreed to develop the piece in phases, and to present and exhibit each step as a different stand-alone integral work within the overall conceptual framework. Largely because of Peter's influence, the piece soon came to focus on the complex cellular interactions within the immune system, with the core engine being a specially written version of his artificial immune system software.

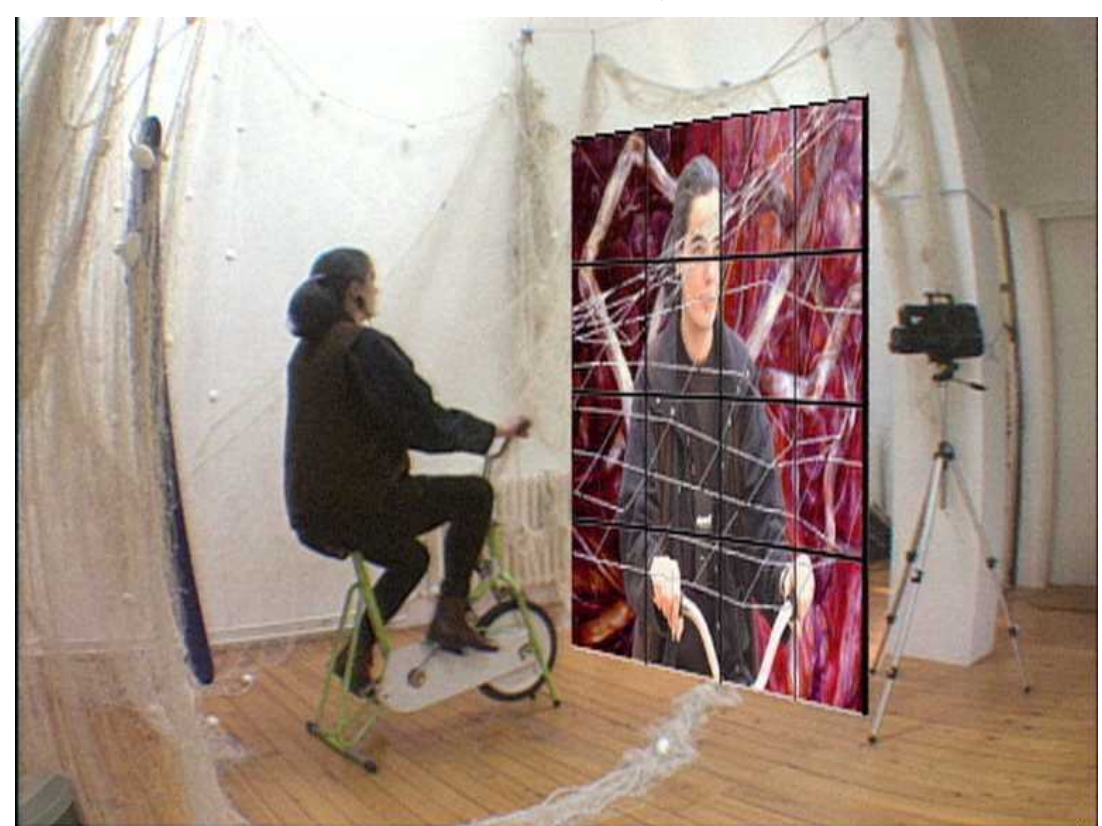

Figure 1. Under the Shirt of a Happy Man (interactive installation, 1994-96)

Algorithmica then evolved into Fugue [12], a project with two potentially conflicting goals: creating an artwork, and developing an audiovisualization of the immune system for scientists. I had not previously been particularly interested in scientific visualisation and had very little insight into the field, but I found it quite surprising that audiovisualisations were very rare in science - in fact, I had only come across one example at the time [5]. In my view, audiovisualisation offers significant advantages for understanding complex systems, because, as Dombois notes, it offers a much wider bandwidth than vision alone, and engages both serial and parallel modes of perception. I was also keen to explore the potential of interactive technologies for enabling users to engage with the production of phenomena, rather than merely observing them passively.
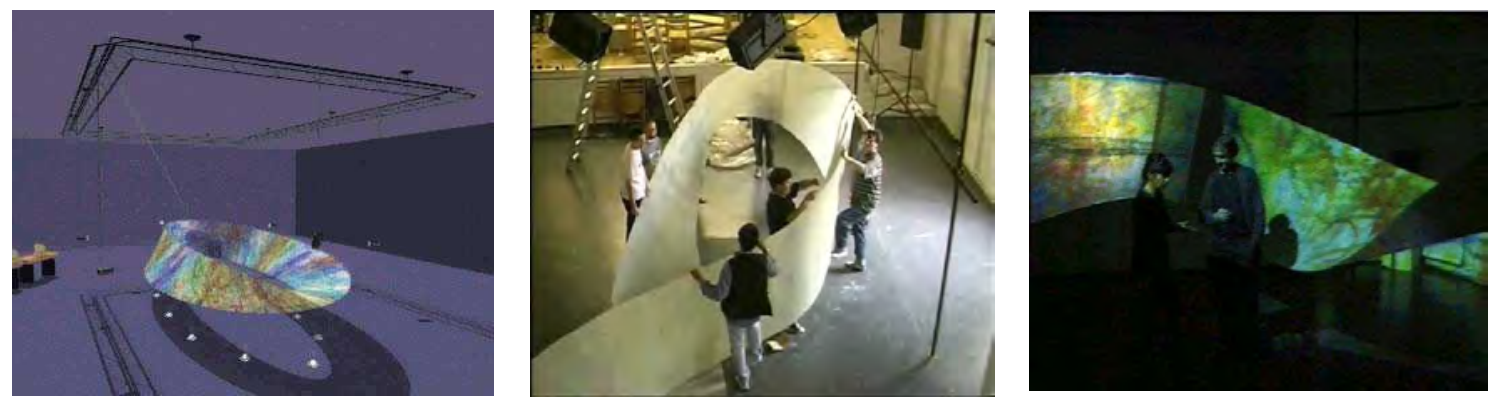

Figure 2. Infonoise (interactive installation, 1998 - 2001)

Left: 3D simulation. Centre: Building the installation. Right: Inside the installation. 
We decided that our focus in Fugue would be on understanding and applying the principles of biological processes, rather than creating photo-realistic 'beautiful imagery', or merely re-representing scientific findings as visualisations or sonifications. My friend, the immunologist Dr Nada Pejnović, a research fellow at St. Mary's Hospital, gave me a detailed introduction to the field, and I was able to discuss with her my artistic interpretations of scientific subjects, my sketches inspired by medical sources, and the concept for the eventual piece. Real-time generated images originating in computational processes would set the framework for the visuals, with a major practical requirement being to reduce the typically heavy computation to a minimum. To meet this condition, and to achieve an abstract, symbolic representation of the actors in the immune system drama, I looked back to the cell-like egg-shaped and spherical structures that had appeared in my paintings from the 1990s and I suggested making some clay models, as I had done for my early paintings. Anthony scanned the clay models in 3D as the starting point for the final look of the inhabitants of our virtual immune system. To emphasise the focus on processes, and the distinction from the aesthetics of the gaming industry and commercial computer graphics, I suggested a black-and-white approach. Both scientists found this idea problematic, because, in their own words, they 'could think of the immune system only in red'. As a compromise, our first prototype was indeed monochromatic, but in red.

The combination of Anthony's expertise in creating 3D wire-frame models of the human body, his taste for abstract visual art, and my experience as a trained painter of using techniques of perspective, colour and sfumato to suggest depth, and to upgrade the crudeness of the computer generated image with the attributes of traditional visual aesthetics, gave rise to an enjoyable creative process. We replaced the red with a greyscale approach, which was now accepted as being congruent with the overall conceptual framework. To complete the basic system, Rainer designed the sound software around a series of customised audio players that he called Fugue Players, which responded in real time to changes within the artificial immune system.

The first outcome of the team's collaboration was a paper [2] presented at a scientific conference. However, our concept received little attention, as most of the scientists categorised it as 'non-scientific', although a small number praised its fresh approach. It is only now as this text is going to press that we have noticed some interest from those dealing with scientific representations, but it is too early to know where this might lead.

\section{VARIATIONS ON THE THEME OF INTERACTIVITY}

After this encounter with scientists, we concentrated on developing the artwork. At the time, I wrote: 'The title Fugue is a metaphor for the transdisciplinary nature of the work, and for the method applied: inter-weaving the different perspectives of artists and scientists. The emergent, evolving nature of the artificial immune system algorithm, the use of repetition in the form of a succession of variations of immune system 'events', and the complex structural and functional interrelationships between the individual elements and processes are strongly related to the musical form of counterpoint, which formed one of the inspirations for the artistic concept for Fugue. The Artificial Immune System software creates the dynamics of the virtual immune system drama, and also 
constructs and implements the architecture of the Fugue by providing the functional structure for the communication channels between the visuals and the sound.

We were engaged in intensive online work shaping the architecture and aesthetics of the installation. Online communication imposes numerous limitations, from the inevitable time lag, to the lack of direct face-to-face discussion. After a period of excitement and enthusiasm, we entered a phase when tensions ran high. This was caused by conflicts between the demand for scientific accuracy, and the artistic interpretation of scientific data, and it escalated to a level that threatened to end the collaboration, and the project. It took a lot of effort from all of us to reach a consensus, and we decided from then on to limit our comments to our own area of expertise.

Rainer and I had conceptualised the interactive component of Fugue as a dialogue between the rhythms of the piece and the biological rhythms of the participants between the computational model and the living body. The engineer Zoran Milković designed the sculptural form for the set - a screen system shaped like a truncated hexagonal pyramid, and large enough to contain several participants, with the visuals being back-projected on to three adjacent walls to achieve an immersive effect. Zoran also produced a 3D computer model that served both as a virtual maquette for designing the interaction software, and as a guide for the final production. This brought a much needed new impetus. Richard Newcombe, a University of Essex computer scientist now at Imperial College, designed the hardware and software to support the interactive component of Fugue. The participants were lit from above by an infrared light source, and the resulting images were streamed from an infrared video camera. Richard then modified his particle filtering and computer vision software, originally designed for scientific research, to track the positions and movements of the participants, and to send these data in real time to the immune system algorithm, which would then respond in a complex time-delayed way through the visuals and the sound. After a few more weeks of intense work on the project, developing a common language with our new collaborating scientist in the process, we had our piece fully developed and tested.

The usual venues for art/science projects are of two kinds: a closed circle of specialised events with well informed audiences, which never engage with the wider art scene; or science education events, which usually target a very young audience and address scientific issues in a predominantly pedagogical manner. The first large-scale interactive Fugue installation (figure 3) in Belgrade in August 2006 was rather different, as it took place in the ULUS fine art gallery. Exhibiting the piece in a fine arts context produced both expected and unexpected results. The expected responses came mainly from artists and scientists, each group initially seeing the piece as the province of the other - a perennial problem with art/science. What was quite unexpected was the degree of suspicion and fear showed by some members of the public, who hesitated at the threshold of the enclosure and refused to enter it, demanding to know what personal data were being collected from them, on whose behalf, and for what purpose. The causes of the intensity of their reaction, and their suspicion of the medical establishment, has become a topic for investigation in future developments of Fugue. 


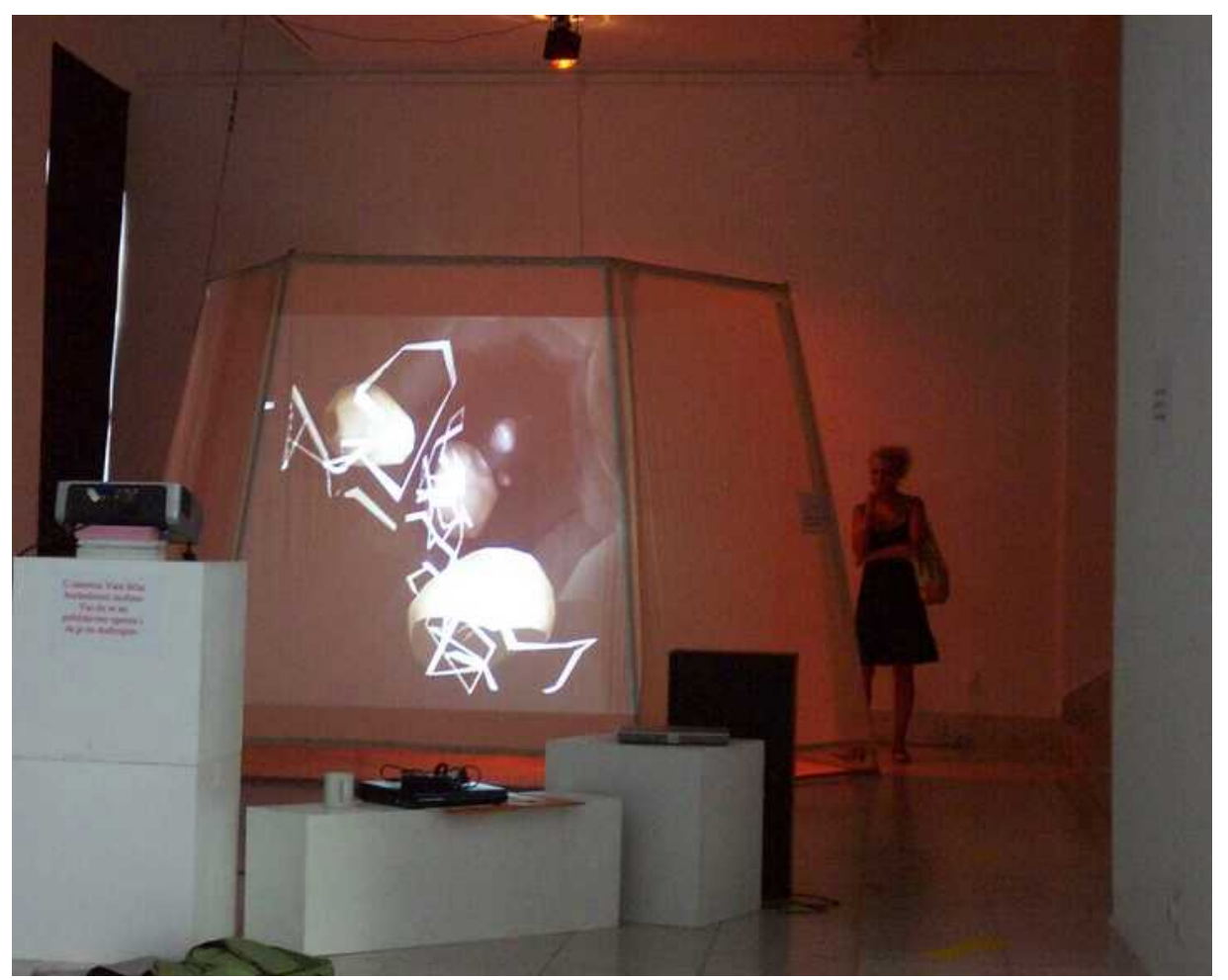

Figure 3. A participant is about to enter the Belgrade installation.

\section{VARIATIONS ON THE THEME OF IMMERSION}

Fugue is currently being exhibited in the large-scale group show entitled Infectious Stay Away at Trinity College Dublin's Science Gallery. Oddly enough, it is the first time that Fugue has been shown in a public engagement context. The curatorial concept for this intriguing exhibition is marked by spectacular design and an imaginative theatrical script. An excellent balance of varied and clearly distinct takes on the theme of infection includes straight scientific demonstrations along with artworks of a more reflective nature, and provides a perfect framework for both the artistic and scientific aspects of the piece to unfold and communicate.

The Science Gallery exhibitions always attract the general public in large numbers (nearly 9,000 in the first week or so of Infectious), and the three month duration of the show demanded a robust, durable and safe design. We therefore decided to show a new version of Fugue, a screen-based presentation of the free-running real-time generated sounds and visuals in a non-interactive and non-immersive environment. For this show, we wanted to guide the audience towards the meditative properties of the piece, and to focus on the complexity of the interplay between the artistic expressions, and the freerunning real-time generated dialogue between the artificial immune system components. We invite visitors to "watch and listen, and become attuned to the processes and rhythms that are mirroring what might be happening right now, inside your own body". The intimate set consisted of a large plasma screen in a small cubicle, juxtaposed with the overall spectacular setting of the gallery and the other pieces. The response from 
both the visitors and the curatorial team has been very positive, with every indication that we have achieved our aims.

\section{QUODLIBET}

Although the content and form of Fugue has attracted most attention, it is perhaps the nature of the technologies employed that carries the deepest message. It is becoming clear that the digital revolution has changed the nature of our perceptual processes, and this in turn has changed our conscious experience of the physical world, inducing changes in cognition on a scale that is still unknown. Some of the most radical insights into the essence of the problems arising from a digital culture come from the media critic Paul Virilio. He identifies the economic and political origins and aspects of the digital revolution, and its socio-political effects, particularly globalisation and global militarization, mediated perception and new forms of alienation. He paints a dark and accurate picture of the current world, with an even darker vision of the future: "One day the day will come when the day won't come" [11]. His disturbing, dramatic warnings about the potential remodelling of humans by means of technology carry a strong message and call for a revolt against the tyranny of real time interactivity and media, questioning the ethics of both the arts and the sciences.

But of course, issues similar to these have been explored across all art disciplines. An increasing number of artists working with technology are investigating and experimenting with the phenomena arising directly from the interplay between our senses and technology - for example, Stelarc through his concepts of obsolete bodies and exoskeletons [9], Char Davis with her pioneering bio-feedback VR [8], Rainer Linz analysing the physiological aspects of electronic music [7], and Margaret Dolinsky using digital art to study cognitive recognition and perceptual shifts [4].

We might think that science should provide some answers to these questions, but with very few exceptions, mainstream science has ignored the possible cognitive consequences of these dramatic environmental changes. For example, the majority of scientists investigating perception, particularly within the discipline of neuroscience, still refer to the 'real world' as a constant, an axiom, unchanged through time. But there is a further problem within neuroscience itself: the dominant doctrine, recognising slow evolutionary processes as the only way for qualitative changes to happen in a species, has combined with the established view that the brain is a closed and static system with fixed locations for particular functions, leaving no space to consider changes occurring within periods of ten years - or even less. It is assumed that we come into this world with a genetically predetermined design for the brain, with exact locations for different functions, and that this structure remains constant throughout our lives.

However, over many years, a small number of scientists have been breaking out of this rigid context to show that the brain is not a closed, unchangeable system. We are now seeing growing scientific evidence that the brain is in fact almost nakedly open to external influences, and is capable of rapid and radical change by remodelling itself through learning and interaction with the environment. The field of neuroscience is now yielding evidence that may revolutionise not only the science of cognition, but also the wider view of the relationship between humans and the environment, and even the role 
and nature of culture. The brain can no longer be regarded as a fixed, closed, passive receiver of information from the senses - a mere processor for the information that controls our body through a kind of one-way communication. Rather, it is intrinsically plastic, in a process of constant change and growth through its interaction with the environment, and through a variety of learning processes.

It is certainly too extensive and complicated a matter to be reviewed adequately in this brief paper, but we can pick out some pioneers. The late Dr Paul Bach-Y-Rita [1] was one of the first neuroscientists to work on what is now called neuroplasticity. His approach was not just theoretical, but practical: he worked with technical experts to construct electronic devices that would enable the brain of a patient with severe sensory problems to recover the lost functions. His method was to provide the patient's brain with the missing information through a different sensory channel. In 1969 he provided blind people with 'visual' information by transferring a camera image to the patients' skin using an array of vibrating pins, and his success led to the radical concept of 'seeing with the brain'.

We also now have a wealth of scientific evidence that shows that the way in which we use and exercise our brains really does matter. Another neuroplasticity pioneer, the neuroscientist Michael Merzenich [15], argues that learning and practising certain skills can rapidly change hundred of millions of connections in our brain, improving and speeding up a wide variety of cognitive abilities. His experiments over the years have delivered strong arguments against the idea of fixed functions in fixed locations in the brain. He has been particularly active in discovering how new learning can stimulate the brain to counteract age-related deterioration, or the effects of serious brain injury, or language impairment in children. Most importantly, he has found that the most powerful way of delivering the learning tasks is through the use of digital technology: the speed and flexibility of his interactive computer-based training scheme enable the delivery of more effective rewards, in turn speeding up the rate of learning.

In a recent book on neuroplasticity, The Brain that Changes Itself, Norman Doidge [3] seems to offer a roadmap for future connections between disciplines grounded in neuroplasticity. In the chapter The Culturally Modified Brain he writes:

As we use an electronic medium, our nervous system extends outwards, and the medium extends inwards. [...] Because our nervous system is plastic, it can take the advantage of this compatibility and merge with the electronic media, making a single, larger system. Indeed, it is the nature of such systems to merge whether they are biological or man-made. [3] p.311.

In the case of an electronic device playing the role of a substitute for a lost capacity, or as an assistant in its regeneration, our body's response can take a dramatic form, because the way in which electronic and digital devices transmit information is in essence quite similar to the basic function of our nervous system - the almost instantaneous transmission of electrical impulses. Due to its capacity for plastic changes, our nervous system easily re-wires itself and makes use of this alternative nervous system. In a passage that could have come from the filmmaker and media critic 
Peter Watkins, Doidge notes that it is actually the form of electronic media, and not so much the content, that affects our cognitive processes:

It is the form of the television medium - cuts, edits, zooms, pans and sudden noises that alters the brain, by activating what Pavlov called the 'orienting response', which occurs whenever we sense a sudden change in the world around us, especially a sudden movement. [...] The response is physiological [...] [3] p.309.

Elsewhere, Merzenich emphasises the unprecedented opportunities that now exist for digital technologies to affect our brains:

The internet is just one of those things that contemporary humans can spend millions of 'practice' events at, that the average human a thousand years ago had absolutely no exposure to. Our brains are massively remodelled by this exposure - but so, too, by reading, by television, by modern electronics, by contemporary music, by contemporary 'tools', etc. [15]

Because we now have this solid evidence that interaction with electronic media not only affects our perception and cognition, but can produce rapid and irreversible changes in our brains, the potentially damaging nature of this aspect of modernity confronts and challenges humanity with a set of serious problems. However, as far as I am aware, the true nature and extent of the influence of the modern urban environment, whether private, public, or workspace, has not yet been the subject of an in-depth scientific analysis. We can, of course, exercise choice even in the face of this onslaught - and neuroplasticity also tells us that the extent to which we can shape our own lives through the ways we choose to use our brains is far larger than we once thought it was.

\section{CODA}

It is clear that as artists we must continue to engage with this theme, although we know that this is a difficult task in this age of immaturity, kindergarten states and the hyperreality of the profit-driven post-digital revolution. For artists, however, there may be problems closer to home. This new understanding of cognitive processes is warning us that experiencing art, and especially electronic art, or technology-enabled art, is far from being an innocently entertaining or aesthetically pleasing experience extending for a limited period of time. The disturbing evidence of neuroplasticity raises the possibility that experiencing particular forms of art may itself affect and mark our cognition perhaps with irreversible and unknown changes. But of course we cannot abandon technology: we must instead seek a deeper understanding of its effects on humanity by looking at all its aspects, positive, negative, and unknown. And here, the dramatic shift in neuroscience brings with it a fascinating opportunity to explore and analyse the effects of electronic media through scientifically informed art, which could give rise to an entirely new art form: neuroplastic art [14].

The concept of neuroplastic art opens a future for scientifically articulate artists and artistically articulate scientists to work closely together, with a full awareness of both 
the potential and the danger that emerges from the parallels between the nature of our nervous system and the characteristics of digital technology and electronic media. It may be possible to structure art works according to new scientific evidence, and to fuse scientific knowledge with imagination to exploit the nature of electronic media to create platforms for experiences that have never existed before. Bringing together the scientists' knowledge about the brain, and our knowledge of the properties of electronic media, we can envisage art works that will become in a way tuneable complex instruments, serving both art and science. Only then will imagination and creativity transcend today's mere fascination with state-of-the-art technology, and use both technology and brain science as a means to express ideas. Perhaps this will even uncover new and benign ways of linking our brains with, and through, technology...

We cannot reverse or stop the process, but we can certainly contribute to an informed awareness of the issues, and we may be able to develop techniques for reducing at least some of the damaging effects. At present, I cannot offer any clear description or prescription for these new art forms except to look for the seeds within existing artistic explorations, such as Fugue. Perhaps a better metaphor might be fugu - the Japanese Tiger Blowfish. When properly prepared by a licenced and trained fugu chef, it is an incomparable and expensive delicacy, but when prepared without the appropriate knowledge and training, the dish may prove fatal.

Finally, as a last comment on our responsibility and opportunity to engage in this issue, we should perhaps recall the prophetic words of Heidegger in one of the most frequently analysed philosophical texts on technology:

...essential reflection upon technology and decisive confrontation with it must happen in a realm that is, on the one hand, akin to the essence of technology and, on the other, fundamentally different from it. Such a realm is art. [6]

\section{ACKNOWLEDGEMENTS}

The author gratefully acknowledges the support of the Computer Science Department, University College London; School of Computer Science and Electronic Engineering, University of Essex; Arts Council England; Arts and Humanities Research Council; Leverhulme Trust; Australian Network for Arts and Technology; ULUS (Serbian Association of Fine Arts); and Trinity College Dublin Science Gallery.

\section{References}

[1] BACH-Y-RITA, Paul, TYLER, Mitchel and KACZMAREK, Kurt. Seeing with the brain. International Journal of Human-Computer Interaction. 15(2), 2003. pp.285-295.

[2] BENTLEY, P.J., NOVAKOVIC, G., and RUTO, A. Fugue : an interactive immersive audiovisualisation and artwork using an artificial immune system. In : Proceedings $4^{\text {th }}$ International Conference, ICARIS 2005, Banff, Alberta, 14-17 August, 2005. Springer, 2005. pp.1-12. Available at: http://dx.doi.org/10.1007/11536444_1

[3] DOIDGE, Norman The brain that changes itself. New York : Viking, 2007. 
[4] DOLINSKY, Margaret. from http://dolinsky.fa.indiana.edu/

[5] DOMBOIS, F. Using audification in planetary seismology. In : Proceedings of the 2001 International Conference on Auditory Display, Espoo, 2001.

[6] HEIDEGGER, Martin. The questions concerning technology, and other essays. New York : Harper Perennial, 1982.

[7] LINZ, Rainer. from http://www.rainerlinz.net/NMA/articles/altering.html

[8] MCROBERT, Laurie. Char Davies' immersive virtual art and the essence of spatiality. University of Toronto Press, 2007.

[9] SMITH, Marquard (Editor) Stelarc : the monograph. Boston : MIT Press, 2007.

[10] NOVAKOVIC, Gordana. Electronic cruelty. In : Engineering nature : art and consciousness in the post-biological era. Edited by Roy Ascott. Bristol : Intellect, 2006.

[11] VIRILIO, Paul. Open Sky. London : Verso, 1995.

[12] http//www.fugueart.com [nd]

[13] http//www.infonoise.net [nd]

[14] http://www.neuroplasticarts.org/ [nd]

[15] http://www.positscience.com/science/global team/merzenich.php [nd] 